\title{
ON THE GEOMETRIC GROWTH IN A CLASS OF HOMOGENEOUS MULTITYPE MARKOV CHAIN
}

\author{
M. GONZÁLEZ,* \\ R. MARTÍNEZ* AND \\ M. MOTA, ${ }^{* *}$ University of Extremadura
}

\begin{abstract}
In this paper, we investigate the geometric growth of homogeneous multitype Markov chains whose states have nonnegative integer coordinates. Such models are considered in a situation similar to the supercritical case for branching processes. Finally, our general theoretical results are applied to a class of controlled multitype branching process in which the control is random.
\end{abstract}

Keywords: Homogeneous multitype Markov chain; geometric growth; multitype branching process

2000 Mathematics Subject Classification: Primary 60J10

Secondary $60 \mathrm{~J} 80$

\section{Introduction}

One of the problems that arises in the scientific literature on population dynamics is the study of the extinction or the indefinite growth of certain biological (human, animal, cell, etc.) or physical (particle, cosmic ray, etc.) populations. Furthermore, if unlimited growth happens, an interesting question from a practical viewpoint is to determine the growth rate of the population. Traditionally, branching processes have been used to model the evolution of such populations, and geometric growth has been found to occur in a situation known as supercritical (see, for example, [5]).

In this work, we deal with a class of stochastic process related to population dynamics and more general than (homogeneous) branching processes, namely homogeneous multitype Markov chains (HMMCs) in discrete time taking values in the space of vectors with nonnegative integer coordinates. This class of process has been recently considered in [1], where their indefinite growth was studied in detail. As a continuation of this study, we can investigate the conditions that must be imposed on such models in order to obtain a geometric rate of growth. The results of this paper provide some answers to such questions. As may be expected, the conditions obtained are closely related to those that appear in the literature on supercritical homogeneous branching processes.

Mathematically, we consider an $m$-dimensional homogeneous Markov chain, $\{\boldsymbol{Z}(n)\}_{n \geq 0}$, whose states have nonnegative integer coordinates, i.e. $S \subseteq \mathbb{N}_{0}^{m}$, where $S$ is the set of states. From now on, this chain is specifically referred to as an HMMC. As a generalization of homogeneous branching processes, we can use HMMCs to model the evolution of a population

Received 13 April 2005; revision received 27 June 2005.

* Postal address: Departamento de Matemáticas, Facultad de Ciencias, Avda. Elvas s/n, 06071 Badajoz, Spain.

** Email address: mota@unex.es 
in which individuals of $m$ different types coexist. More specifically, the $i$ th coordinate of $\boldsymbol{Z}(n)$ might represent the number of type- $i$ individuals $n$ generations after the process was started.

In the next section, we investigate the limiting behaviour of some sequences of linear functionals associated with HMMCs, showing that, under certain conditions, they can be suitably normalized using a sequence of constants describing geometric growth. In Section 3, we return to the $m$-dimensional process $\{\boldsymbol{Z}(n)\}_{n \geq 0}$ and prove that this process and its associated linear functionals have the same growth rate, considering almost-sure convergence and $L^{\alpha}$-convergence $(1 \leq \alpha \leq 2)$. Finally, in order to illustrate the power of our general theoretical results, in Section 4 they are applied to a class of controlled multitype branching process.

\section{Asymptotic behaviour of $\{Z(n) \mu\}_{n \geq 0}$}

In this section, we investigate the geometric growth of the sequence of linear functionals $\{\boldsymbol{Z}(n) \boldsymbol{\mu}\}_{n \geq 0}$, where $\boldsymbol{\mu} \in \mathbb{R}_{+}^{m}$ is a vector with positive coordinates (and the scalar product is implicit in vector multiplication). Some of these sequences will play an important role in our later study of $\{\boldsymbol{Z}(n)\}_{n \geq 0}$. Although the process $\{\boldsymbol{Z}(n) \boldsymbol{\mu}\}_{n \geq 0}$ is associated to the HMMC $\{\boldsymbol{Z}(n)\}_{n \geq 0}$, notice that this process is not a Markov chain.

We consider a $\boldsymbol{\mu} \in \mathbb{R}_{+}^{m}$ such that

$$
r_{\boldsymbol{\mu}}:=\lim _{\|z\| \rightarrow \infty} \frac{\mathrm{E}[\boldsymbol{Z}(n+1) \boldsymbol{\mu} \mid \boldsymbol{Z}(n)=z]}{z \boldsymbol{\mu}}>1,
$$

where $\|\cdot\|$ denotes an arbitrary norm on $\mathbb{R}^{m}$. Notice that (2.1) is an assumption on the Markov chain $\{\boldsymbol{Z}(n)\}_{n \geq 0}$, and that $r_{\boldsymbol{\mu}}$ can be interpreted as the mean growth rate of the process $\{\boldsymbol{Z}(n) \boldsymbol{\mu}\}_{n \geq 0}$. As we pointed out, this situation corresponds to the supercritical case in the context of branching processes. Throughout the paper, we suppose that $\mathrm{E}\left[Z_{i}(1) \mid \boldsymbol{Z}(0)=\boldsymbol{z}\right]<\infty$, for all $z \in \mathbb{N}_{0}^{m}$ and $i \in\{1, \ldots, m\}$, and $\mathrm{P}[\|\boldsymbol{Z}(n)\| \rightarrow \infty]>0$, in order that the study make sense. Sufficient conditions for the event $\{\|\boldsymbol{Z}(n)\| \rightarrow \infty\}$ to have positive probability, under (2.1), were proposed in [1]. Obviously, since $\boldsymbol{\mu} \in \mathbb{R}_{+}^{m}$, this event can be rewritten as $\{\boldsymbol{Z}(n) \boldsymbol{\mu} \rightarrow \infty\}$.

For each $z \in \mathbb{N}_{0}^{m}$ and $\beta \geq 1$, we introduce the functions $h_{\boldsymbol{\mu}}(\boldsymbol{z})$ and $G_{\boldsymbol{\mu}, \beta}(\boldsymbol{z})$, as follows:

$$
h_{\boldsymbol{\mu}}(z):=\frac{\mathrm{E}[\boldsymbol{Z}(n+1) \boldsymbol{\mu} \mid \boldsymbol{Z}(n)=z]}{z \boldsymbol{\mu}}-r_{\boldsymbol{\mu}} \quad \text { if } z \neq \mathbf{0}, \quad h_{\boldsymbol{\mu}}(\mathbf{0}):=1,
$$

with $\mathbf{0}$ the null vector, and

$$
G_{\boldsymbol{\mu}, \beta}(\boldsymbol{z}):=\left(\mathrm{E}\left[|\boldsymbol{Z}(n+1) \boldsymbol{\mu}-\mathrm{E}[\boldsymbol{Z}(n+1) \boldsymbol{\mu} \mid \boldsymbol{Z}(n)=\boldsymbol{z}]|^{\beta} \mid \boldsymbol{Z}(n)=\boldsymbol{z}\right]\right)^{1 / \beta} .
$$

Notice that $r_{\mu}, h_{\mu}(z)$, and $G_{\mu, \beta}(z)$ depend on the chosen vector $\mu$. However, in the rest of the paper we will avoid using $\boldsymbol{\mu}$ in the notation and will instead write $r, h(z)$, and $G_{\beta}(z)$, respectively, unless there is some ambiguity.

The following result allows us to conclude that, under some conditions, $\left\{r^{n}\right\}_{n \geq 0}$ is the only sequence of constants which can describe geometric growth of the process $\{\boldsymbol{Z}(n) \boldsymbol{\mu}\}_{n \geq 0}$.

Proposition 2.1. Let $\{\boldsymbol{Z}(n)\}_{n \geq 0}$ be an HMMC and choose a $\boldsymbol{\mu} \in \mathbb{R}_{+}^{m}$ satisfying (2.1). If $G_{1+\delta}(z)=O\left(\|z\|^{\delta /(1+\delta)}\right)$ for some $\delta \geq 0$, then

$$
\lim _{n \rightarrow \infty} \frac{\boldsymbol{Z}(n+1) \boldsymbol{\mu}}{\boldsymbol{Z}(n) \boldsymbol{\mu}}=r \text { almost surely (a.s.) on }\{\boldsymbol{Z}(n) \boldsymbol{\mu} \rightarrow \infty\} .
$$


Proof. First, notice that the event $\{\boldsymbol{Z}(n) \boldsymbol{\mu} \rightarrow \infty\}$ has positive probability since (2.1) holds and $G_{1+\delta}(z)=O\left(\|z\|^{\delta /(1+\delta)}\right)$ for some $\delta \geq 0$ (see Theorem 1 of [1]). Therefore, for simplicity and without lost of generality, we can assume that $\mathrm{P}[\boldsymbol{Z}(n) \boldsymbol{\mu} \rightarrow \infty]=1$.

Since $r>1$, we can take an $\varepsilon$ such that $0<\varepsilon<r-1$. Define

$$
B_{n}:=\{|\boldsymbol{Z}(n+1) \boldsymbol{\mu}-r \boldsymbol{Z}(n) \boldsymbol{\mu}|<\varepsilon \boldsymbol{Z}(n) \boldsymbol{\mu}\}, \quad n \geq 0 .
$$

It is then sufficient to prove that

$$
\mathrm{P}\left[\bigcup_{k=0}^{\infty} \bigcap_{n=k}^{\infty} B_{n}\right]=1
$$

For any $N>0$, we define the stopping time $T(N)$ by

$$
T(N):= \begin{cases}\min \{n: \boldsymbol{Z}(n) \boldsymbol{\mu} \geq N\} & \text { if there exists an } n \text { such that } \boldsymbol{Z}(n) \boldsymbol{\mu} \geq N, \\ \infty & \text { if } \boldsymbol{Z}(n) \boldsymbol{\mu}<N \text { for all } n .\end{cases}
$$

Since $\mathrm{P}[\boldsymbol{Z}(n) \boldsymbol{\mu} \rightarrow \infty]=1$, we have $\mathrm{P}[T(N)<\infty]=1$. It also follows that

$$
\mathrm{P}\left[\bigcup_{k=0}^{\infty} \bigcap_{n=k}^{\infty} B_{n}\right]=\sum_{k=0}^{\infty} \mathrm{P}\left[\bigcap_{n=k}^{\infty} B_{n} \mid T(N)=k\right] \mathrm{P}[T(N)=k] .
$$

Moreover, given that $\{\boldsymbol{Z}(n)\}_{n \geq 0}$ is an HMMC, for any $k \geq 0$ we have

$$
\begin{aligned}
\mathrm{P}\left[\bigcap_{n=k}^{\infty} B_{n} \mid T(N)=k\right] & =\mathrm{P}\left[\bigcap_{n=k}^{\infty} B_{n} \mid \boldsymbol{Z}(t) \boldsymbol{\mu}<N, t=0,1, \ldots, k-1, \boldsymbol{Z}(k) \boldsymbol{\mu} \geq N\right] \\
& \geq \inf _{\{z: z \boldsymbol{\mu} \geq N\}} \mathrm{P}\left[\bigcap_{n=k}^{\infty} B_{n} \mid \boldsymbol{Z}(k)=\boldsymbol{z}\right]
\end{aligned}
$$

and, taking into account the fact that $\sum_{k=0}^{\infty} \mathrm{P}[T(N)=k]=1$, from (2.3) and (2.4) we find that

$$
\mathrm{P}\left[\bigcup_{k=0}^{\infty} \bigcap_{n=k}^{\infty} B_{n}\right] \geq \inf _{\{z: z \mu \geq N\}} \mathrm{P}\left[\bigcap_{n=0}^{\infty} B_{n} \mid \boldsymbol{Z}(0)=z\right] .
$$

To complete the proof, it is enough to show that

$$
\lim _{\|z\| \rightarrow \infty} \mathrm{P}\left[\bigcup_{n=0}^{\infty} B_{n}^{\mathrm{c}} \mid \boldsymbol{Z}(0)=z\right]=0 .
$$

Indeed, if (2.6) holds then, for every $\eta>0$, there exists an $N \equiv N(\eta)>0$ such that

$$
\mathrm{P}\left[\bigcap_{n=0}^{\infty} B_{n} \mid \boldsymbol{Z}(0)=z\right] \geq 1-\eta
$$

for all $z$ such that $z \boldsymbol{\mu} \geq N$. By taking (2.5) into account, we then find that

$$
\mathrm{P}\left[\bigcup_{k=0}^{\infty} \bigcap_{n=k}^{\infty} B_{n}\right] \geq 1-\eta
$$

for all $\eta>0$. Therefore, (2.2) holds. 
Let us prove (2.6). We rewrite $\bigcup_{n=0}^{\infty} B_{n}^{\mathrm{c}}$ as a disjoint union of sets $C_{n}$, defined as follows:

$$
C_{0}:=B_{0}^{\mathrm{c}}, \quad C_{n}:=B_{n}^{\mathrm{c}} \cap B_{n-1} \cap \cdots \cap B_{0}, \quad n \geq 1 .
$$

Thus,

$$
\mathrm{P}\left[\bigcup_{n=0}^{\infty} B_{n}^{\mathrm{c}} \mid \boldsymbol{Z}(0)=z\right]=\sum_{n=0}^{\infty} \mathrm{P}\left[C_{n} \mid \boldsymbol{Z}(0)=z\right] .
$$

Let us properly bound $\mathrm{P}\left[C_{n} \mid \boldsymbol{Z}(0)=\boldsymbol{z}\right]$, at least for $\boldsymbol{z}$ such that $\|\boldsymbol{z}\|$ is greater than or equal to some constant $k_{1}$. Since $B_{0} \cap \cdots \cap B_{n-1} \in \mathcal{F}_{n}$, where $\mathcal{F}_{n}:=\sigma(\boldsymbol{Z}(0), \ldots, \boldsymbol{Z}(n))$ for $n \geq 0$, it follows that

$$
\begin{aligned}
\mathrm{P}\left[C_{n} \mid \boldsymbol{Z}(0)=z\right] & =\mathrm{E}\left[1_{B_{n}^{\mathrm{c}} \cap B_{n-1} \cap \cdots \cap B_{0}} \mid \boldsymbol{Z}(0)=\boldsymbol{z}\right] \\
& =\mathrm{E}\left[1_{B_{0} \cap \cdots \cap B_{n-1}} \mathrm{E}\left[1_{B_{n}^{\mathrm{c}}} \mid \mathcal{F}_{n}\right] \mid \boldsymbol{Z}(0)=\boldsymbol{z}\right],
\end{aligned}
$$

where $1_{B}$ is the indicator function of the set $B$. Since $(z \boldsymbol{\mu})^{-1} \mathrm{E}[\boldsymbol{Z}(n+1) \boldsymbol{\mu} \mid \boldsymbol{Z}(n)=\boldsymbol{z}]$ converges to $r$ as $\|z\| \rightarrow \infty$, there exists a constant $k_{1}>0$ such that, for all $\tilde{z}$ with $\|\tilde{z}\| \geq k_{1}$,

$$
\left|(\tilde{z} \boldsymbol{\mu})^{-1} \mathrm{E}[\boldsymbol{Z}(n+1) \boldsymbol{\mu} \mid \boldsymbol{Z}(n)=\tilde{\boldsymbol{z}}]-r\right|<\frac{1}{2} \varepsilon .
$$

Also, since $G_{1+\delta}(z)=O\left(\|z\|^{\delta /(1+\delta)}\right)$, there exists a constant $A>0$ such that $G_{1+\delta}(\tilde{z}) \leq$ $A(\tilde{z} \boldsymbol{\mu})^{\delta /(1+\delta)}$. Thus, if $\|\tilde{z}\| \geq k_{1}$ then, by applying Markov's inequality, we conclude that

$$
\begin{aligned}
\mathrm{P}\left[B_{n}^{\mathrm{c}} \mid \boldsymbol{Z}(n)=\tilde{\boldsymbol{z}}\right] & =\mathrm{P}[|\boldsymbol{Z}(n+1) \boldsymbol{\mu}-r \boldsymbol{Z}(n) \boldsymbol{\mu}| \geq \varepsilon \boldsymbol{Z}(n) \boldsymbol{\mu} \mid \boldsymbol{Z}(n)=\tilde{\boldsymbol{z}}] \\
& \leq \mathrm{P}\left[|\boldsymbol{Z}(n+1) \boldsymbol{\mu}-\mathrm{E}[\boldsymbol{Z}(n+1) \boldsymbol{\mu} \mid \boldsymbol{Z}(n)]| \geq \frac{1}{2}(\boldsymbol{Z}(n) \boldsymbol{\mu}) \varepsilon \mid \boldsymbol{Z}(n)=\tilde{\boldsymbol{z}}\right] \\
& \leq(2 A)^{1+\delta} \varepsilon^{-(1+\delta)}(\tilde{\boldsymbol{z}} \boldsymbol{\mu})^{-1} \\
& =C(\tilde{\boldsymbol{z}} \boldsymbol{\mu})^{-1}
\end{aligned}
$$

where $C:=(2 A)^{1+\delta} \varepsilon^{-(1+\delta)}$. Moreover, since $r-\varepsilon>1$,

$$
B_{0} \cap \cdots \cap B_{n-1} \subseteq\left\{\boldsymbol{Z}(n) \boldsymbol{\mu} \geq(r-\varepsilon)^{n} \boldsymbol{Z}(0) \boldsymbol{\mu}\right\},
$$

and there exists a $k_{2}>0$ such that if $\tilde{z} \boldsymbol{\mu}>k_{2}$ then $\|\tilde{z}\| \geq k_{1}$, from (2.7) and (2.8) we find that if $\boldsymbol{z} \boldsymbol{\mu}>k_{2}$ then

$$
\mathrm{P}\left[C_{n} \mid \boldsymbol{Z}(0)=z\right] \leq C(z \boldsymbol{\mu})^{-1}(r-\varepsilon)^{-n}
$$

and, thus,

$$
\mathrm{P}\left[\bigcup_{n=0}^{\infty} B_{n}^{\mathrm{c}} \mid \boldsymbol{Z}(0)=z\right] \leq C(z \mu)^{-1} \sum_{n=0}^{\infty}(r-\varepsilon)^{-n}
$$

converges to 0 as $\|z\| \rightarrow \infty$ (because $r-\varepsilon>1$ ), which concludes the proof.

Define $W(n):=r^{-n} \boldsymbol{Z}(n) \boldsymbol{\mu}$ for all $n \geq 0$. Our objective is to investigate sufficient conditions for the almost-sure convergence and the $L^{\alpha}$-convergence $(1 \leq \alpha \leq 2)$ of the process $\{W(n)\}_{n \geq 0}$ to a finite random variable nondegenerate at 0 . To this end, two important facts must be taken into account.

(i) The expectation $\mathrm{E}[Z(1) \boldsymbol{\mu} \mid \boldsymbol{Z}(0)=\mathbf{0}]$ may be positive, i.e. $\mathbf{0}$ is not necessarily an absorbing state. In addition, the condition $\mathrm{E}[Z(1) \boldsymbol{\mu} \mid \boldsymbol{Z}(0)=\mathbf{0}]<\infty$ means that the expected immigration at the state $\mathbf{0}$ is at most finite. 
(ii) For almost every $\omega \in\{\|\boldsymbol{Z}(n)\| \rightarrow \infty\}$, there exists an $n_{0} \equiv n_{0}(\omega)$ such that $\boldsymbol{Z}(n)(\omega) \boldsymbol{\mu}$ is greater than 0 for every $n \geq n_{0}$. Moreover, if $\mathbf{0}$ is an absorbing state then $\{\boldsymbol{Z}(n) \boldsymbol{\mu}\}_{n \geq 0}$ is positive on the set $\{\|\boldsymbol{Z}(n)\| \rightarrow \infty\}$.

The methodology applied in the proofs of the following results is similar to that applied in [3] in the context of population-size-dependent branching processes.

Our first step is to establish a condition that guarantees the convergence of the sequence $\{\mathrm{E}[W(n)]\}_{n \geq 0}$ to a finite and positive limit. This condition depends on the speed of the convergence in (2.1), which is determined by the function $h(z)$ and is given in terms of the norm one, that is $\|\boldsymbol{z}\|=\boldsymbol{z} \mathbf{1}$, where $\mathbf{1}$ is an $m$-dimensional vector with all its coordinates equal to 1 .

Theorem 2.1. Let $\{\boldsymbol{Z}(n)\}_{n \geq 0}$ be an HMMC such that $\mathrm{P}[\|\boldsymbol{Z}(n)\| \rightarrow \infty]>0$, and choose a $\mu \in \mathbb{R}_{+}^{m}$ satisfying (2.1). Suppose that there exists a nonincreasing sequence $\{\bar{h}(n)\}_{n \geq 1}$ such that, for all non-null vectors $\boldsymbol{z},|h(z)| \leq \bar{h}(\boldsymbol{z} \mathbf{1})$ and $\sum_{n=1}^{\infty} n^{-1} \bar{h}(n)<\infty$. Then

$$
0<\lim _{n \rightarrow \infty} \mathrm{E}[W(n)]<\infty .
$$

Proof. First, let us prove that the limit of the sequence $\{\mathrm{E}[W(n)]\}_{n \geq 0}$ exists. Since $\mathrm{P}[\boldsymbol{Z}(n) \boldsymbol{\mu} \rightarrow \infty]>0$, it is possible to find an $n_{0} \geq 0$ such that $\mathrm{E}[W(n)]>0$ for all $n \geq n_{0}$.

Taking into account the definition of the function $h(z)$, we deduce that

$$
\boldsymbol{Z}(n) \boldsymbol{\mu} h(\boldsymbol{Z}(n))=\mathrm{E}[\boldsymbol{Z}(n+1) \boldsymbol{\mu} \mid \boldsymbol{Z}(n)]-r \boldsymbol{Z}(n) \boldsymbol{\mu} \quad \text { a.s. on }\{\boldsymbol{Z}(n) \neq \mathbf{0}\} .
$$

By applying Lemma A.2 (see Appendix A) to the sequence $\{\bar{h}(n)\}_{n \geq 1}$, we find that there exists a nonincreasing positive function $\bar{h}_{1}(x), x \in \mathbb{R}_{+}$, such that $x \bar{h}_{1}(x)$ is concave and nondecreasing, $\bar{h}(n) \leq \bar{h}_{1}(n)$ for all $n \geq 1$, and $\sum_{n=1}^{\infty} n^{-1} \bar{h}_{1}(n)<\infty$. If $n \geq n_{0}$ then, using Jensen's inequality, we have

$$
\begin{aligned}
|\mathrm{E}[W(n+1)]-\mathrm{E}[W(n)]|= & r^{-(n+1)}|\mathrm{E}[\boldsymbol{Z}(n+1) \boldsymbol{\mu}-r \boldsymbol{Z}(n) \boldsymbol{\mu}]| \\
\leq & r^{-(n+1)} \mathrm{E}\left[|\mathrm{E}[\boldsymbol{Z}(n+1) \boldsymbol{\mu} \mid \boldsymbol{Z}(n)]-r \boldsymbol{Z}(n) \boldsymbol{\mu}| 1_{\{\boldsymbol{Z}(n) \neq \mathbf{0}\}}\right] \\
& +r^{-(n+1)} \mathrm{E}\left[\mathrm{E}[\boldsymbol{Z}(n+1) \boldsymbol{\mu} \mid \boldsymbol{Z}(n)] 1_{\{\boldsymbol{Z}(n)=\mathbf{0}\}}\right] \\
= & r^{-1} \mathrm{E}\left[|W(n) h(\boldsymbol{Z}(n))| 1_{\{\boldsymbol{Z}(n) \neq \mathbf{0}\}}\right]+\mathrm{E}[W(n+1) \mid \boldsymbol{Z}(n)=\mathbf{0}] \\
\leq & r^{-1} \mathrm{E}\left[W(n) \bar{h}_{1}(\boldsymbol{Z}(n) \mathbf{1})\right]+C_{1} r^{-n} \\
\leq & C_{2} \mathrm{E}[W(n)] \bar{h}_{1}(\mathrm{E}[\boldsymbol{Z}(n) \mathbf{1}])+C_{1} r^{-n} \\
\leq & \mathrm{E}[W(n)]\left(C_{2} \bar{h}_{1}\left(r^{n} C_{3} \mathrm{E}[W(n)]\right)+C_{1} r^{-n} \mathrm{E}[W(n)]^{-1}\right),
\end{aligned}
$$

for some constants $C_{1}, C_{2}, C_{3}>0$. Since $\{\mathrm{E}[W(n)]\}_{n \geq n_{0}}$ and $f(x):=C_{2} \bar{h}_{1}\left(C_{3} x\right)+C_{1} x^{-1}$ satisfy the assumptions of Lemma A.3, there exists an

$$
L:=\lim _{n \rightarrow \infty} \mathrm{E}[W(n)]<\infty .
$$

Moreover, we can find a value $c_{0}$ such that if $\mathrm{E}[W(n)]>c_{0}$ for some $n \geq n_{0}$, then $L$ is positive.

In particular, we have proved that, for every state $\hat{z} \in \mathbb{N}_{0}^{m}$ such that $\hat{z} \boldsymbol{\mu}>c_{0}$ and $\mathrm{P}[\boldsymbol{Z}(n) \boldsymbol{\mu} \rightarrow \infty \mid \boldsymbol{Z}(0)=\hat{\boldsymbol{z}}]>0$, we have

$$
\lim _{n \rightarrow \infty} \mathrm{E}[W(n) \mid \boldsymbol{Z}(0)=\hat{z}]>0
$$


Since $\mathrm{P}[\boldsymbol{Z}(n) \boldsymbol{\mu} \rightarrow \infty]>0$ and

$$
\{\boldsymbol{Z}(n) \boldsymbol{\mu} \rightarrow \infty\}=\bigcup_{n=0}^{\infty} \bigcap_{k=n}^{\infty}\left(\left\{\boldsymbol{Z}(k) \boldsymbol{\mu}>c_{0}\right\} \cap\{\boldsymbol{Z}(n) \boldsymbol{\mu} \rightarrow \infty\}\right),
$$

there exist a $\hat{z} \in \mathbb{N}_{0}^{m}$ and an $n_{1} \geq 0$ such that $\hat{z} \boldsymbol{\mu}>c_{0}$,

$$
\mathrm{P}\left[\boldsymbol{Z}\left(n_{1}\right)=\hat{z}\right]>0, \quad \text { and } \quad \mathrm{P}[\boldsymbol{Z}(n) \boldsymbol{\mu} \rightarrow \infty \mid \boldsymbol{Z}(0)=\hat{z}]>0 .
$$

Now, using (2.9) and some known results on Markov chains, it can be shown that, for all $n \geq n_{1}$,

$$
\mathrm{E}[W(n)]=r^{-n_{1}} \sum_{\tilde{z} \in \mathbb{N}_{0}^{m}} \mathrm{P}\left[\boldsymbol{Z}\left(n_{1}\right)=\tilde{z}\right] \mathrm{E}\left[W\left(n-n_{1}\right) \mid \boldsymbol{Z}(0)=\tilde{z}\right]
$$

and, therefore,

$$
\lim _{n \rightarrow \infty} \mathrm{E}[W(n)] \geq r^{-n_{1}} \mathrm{P}\left[\boldsymbol{Z}\left(n_{1}\right)=\hat{z}\right] \lim _{n \rightarrow \infty} \mathrm{E}\left[W\left(n-n_{1}\right) \mid \boldsymbol{Z}(0)=\hat{z}\right]>0,
$$

which concludes the proof.

In the following result, we obtain a sufficient condition for the almost-sure convergence of the sequence $\{W(n)\}_{n \geq 0}$.

Proposition 2.2. Let $\{\boldsymbol{Z}(n)\}_{n \geq 0}$ be an HMMC such that $\mathrm{P}[\|\boldsymbol{Z}(n)\| \rightarrow \infty]>0$, and choose a $\boldsymbol{\mu} \in \mathbb{R}_{+}^{m}$ satisfying (2.1). Suppose that there exists a nonincreasing sequence $\{\bar{h}(n)\}_{n \geq 1}$ such that, for all non-null vectors $\boldsymbol{z},|h(\boldsymbol{z})| \leq \bar{h}(\boldsymbol{z} \mathbf{1})$ and $\sum_{n=1}^{\infty} n^{-1} \bar{h}(n)<\infty$. Then there exists a nonnegative, finite random variable $W$ such that $\{W(n)\}_{n \geq 0}$ converges almost surely to $W$.

Proof. We define the sequences $\{T(n)\}_{n \geq 0}$ and $\{Y(n)\}_{n \geq 0}$ as follows:

$$
\begin{gathered}
T(0):=0, \quad T(n):=r^{-1} \sum_{k=0}^{n-1} W(k) h(\boldsymbol{Z}(k)), \quad n>0, \\
Y(n):=W(n)-T(n), \quad n \geq 0 .
\end{gathered}
$$

Given the previous relation, it is enough to prove that both sequences converge almost surely.

Let us first prove that the sequence $\{T(n)\}_{n \geq 0}$ converges almost surely to a finite limit. By taking a function $\bar{h}_{1}(x)$ as in the proof of Theorem 2.1 and applying Jensen's inequality, we have

$$
\begin{aligned}
\mathrm{E}[|W(n) h(\boldsymbol{Z}(n))|] & \leq C_{1} \mathrm{E}[W(n)] \bar{h}_{1}(\mathrm{E}[\boldsymbol{Z}(n) \mathbf{1}]) \\
& \leq C_{1} \mathrm{E}[W(n)] \bar{h}_{1}\left(r_{\mu}^{n} C_{2} \mathrm{E}[W(n)]\right)
\end{aligned}
$$

for some constants $C_{1}, C_{2}>0$. Moreover, from Theorem 2.1 we find that

$$
0<\lim _{n \rightarrow \infty} \mathrm{E}[W(n)]<\infty,
$$

and, by taking into account the fact that $\sum_{n=1}^{\infty} n^{-1} \bar{h}_{1}(n)<\infty$, it follows that

$$
\mathrm{E}\left[\sum_{n=0}^{\infty} W(n)|h(\boldsymbol{Z}(n))|\right] \leq \sum_{n=0}^{\infty} C_{1} \mathrm{E}[W(n)] \bar{h}_{1}\left(r_{\mu}^{n} C_{2} \mathrm{E}[W(n)]\right)<\infty .
$$


Thus, $\sum_{n=0}^{\infty} W(n) h(\boldsymbol{Z}(n))$ converges almost surely, which implies that the sequence $\{T(n)\}_{n \geq 0}$ also converges almost surely.

Finally, let us prove that the sequence $\{Y(n)\}_{n \geq 0}$ is a submartingale with respect to the sequence of $\sigma$-algebras $\left\{\mathcal{F}_{n}\right\}_{n \geq 0}$. Indeed, on $\{\boldsymbol{Z}(n) \neq \mathbf{0}\}$ we have

$$
\begin{aligned}
\mathrm{E}\left[Y(n+1) \mid \mathcal{F}_{n}\right] & =\mathrm{E}\left[W(n+1) \mid \mathcal{F}_{n}\right]-T(n+1) \\
& =W(n)+r^{-1} W(n) h(\boldsymbol{Z}(n))-T(n+1) \\
& =Y(n) \quad \text { a.s., }
\end{aligned}
$$

and on $\{\boldsymbol{Z}(n)=\mathbf{0}\}$ we have

$$
\begin{aligned}
\mathrm{E}\left[Y(n+1) \mid \mathcal{F}_{n}\right] & =\mathrm{E}[W(n+1) \mid \boldsymbol{Z}(n)=\mathbf{0}]-T(n) \\
& \geq-T(n) \\
& =Y(n) \quad \text { a.s. }
\end{aligned}
$$

Since $Y(n)=W(n)-T(n),(2.10)$ holds, and $\lim _{n \rightarrow \infty} \mathrm{E}[W(n)]<\infty$, it follows that

$$
\sup _{n \geq 0} \mathrm{E}[|Y(n)|]<\infty
$$

Hence, by applying the martingale convergence theorem, we can ensure that the sequence $\{Y(n)\}_{n \geq 0}$ converges almost surely to a finite limit, and the proof is complete.

Obviously $W$ can be non-null only on the set $\{\boldsymbol{Z}(n) \boldsymbol{\mu} \rightarrow \infty\}$, that is, $\{W>0\} \subseteq$ $\{\boldsymbol{Z}(n) \boldsymbol{\mu} \rightarrow \infty\}$. In the following result, we propose conditions (which obviously include the previous ones) for the variable $W$ to be nondegenerate at 0 . In fact, under these conditions we obtain the $L^{\alpha}$-convergence of the sequence $\{W(n)\}_{n \geq 0}$ for $1 \leq \alpha \leq 2$.

Theorem 2.2. Let $\{\boldsymbol{Z}(n)\}_{n \geq 0}$ be an HMMC such that $\mathrm{P}[\|\boldsymbol{Z}(n)\| \rightarrow \infty]>0$, and choose a $\boldsymbol{\mu} \in \mathbb{R}_{+}^{m}$ satisfying (2.1). Also let $1 \leq \alpha \leq 2$ and suppose that there exist sequences $\{\bar{h}(n)\}_{n \geq 1}$ and $\left\{\bar{g}_{\alpha}(n)\right\}_{n \geq 1}$ such that

(i) for each non-null vector $z,|h(z)| \leq \bar{h}(z \mathbf{1})$ and $G_{\alpha}(z) \leq \bar{g}_{\alpha}(z \mathbf{1})$; and

(ii) the sequences $\{\bar{h}(n)\}_{n \geq 1}$ and $\left\{n^{-1} \bar{g}_{\alpha}(n)\right\}_{n \geq 1}$ are nonincreasing and

$$
\sum_{n=1}^{\infty} n^{-1} \bar{h}(n)<\infty, \quad \sum_{n=1}^{\infty} n^{-2} \bar{g}_{\alpha}(n)<\infty .
$$

Then $\{W(n)\}_{n \geq 0}$ converges almost surely and in $L^{\alpha}$ to a finite random variable $W$ that is nondegenerate at 0 .

Proof. Taking into account the properties of the sequence $\{\bar{h}(n)\}_{n \geq 1}$, Theorem 2.1, and Proposition 2.2, it is enough to show that the sequence $\{W(n)\}_{n \geq 0}$ converges in $L^{\alpha}$ to a limit $W$. Therefore, we prove that the sequence $\{W(n)\}_{n \geq 0}$ satisfies the $L^{\alpha}$-Cauchy convergence criterion. Indeed, if we denote by $\|\cdot\|_{\alpha}$ the norm on $L^{\alpha}$, we obtain the following inequality:

$$
\begin{aligned}
\|\boldsymbol{Z}(n+1) \boldsymbol{\mu}-r \boldsymbol{Z}(n) \boldsymbol{\mu}\|_{\alpha} \leq & \|\boldsymbol{Z}(n+1) \boldsymbol{\mu}-\mathrm{E}[\boldsymbol{Z}(n+1) \boldsymbol{\mu} \mid \boldsymbol{Z}(n)]\|_{\alpha} \\
& +\|\mathrm{E}[\boldsymbol{Z}(n+1) \boldsymbol{\mu} \mid \boldsymbol{Z}(n)]-r \boldsymbol{Z}(n) \boldsymbol{\mu}\|_{\alpha} .
\end{aligned}
$$


Owing to the properties of the sequence $\{\bar{h}(n)\}_{n \geq 1}$, Lemma A.2 guarantees the existence of a nonincreasing function $\hat{h}(x)$ such that $\bar{h}(n) \leq \hat{h}(n), x \hat{h}^{\alpha}\left(x^{1 / \alpha}\right)$ is concave, and $\int_{1}^{\infty} x^{-1} \hat{h}(x) \mathrm{d} x$ $<\infty$. By applying Jensen's inequality, we find that there exist constants $C_{1}, C_{2}>0$ such that

$$
\begin{aligned}
\mathrm{E}\left[|\mathrm{E}[\boldsymbol{Z}(n+1) \boldsymbol{\mu} \mid \boldsymbol{Z}(n)]-r \boldsymbol{Z}(n) \boldsymbol{\mu}|^{\alpha}\right] \leq & \mathrm{E}\left[(\boldsymbol{Z}(n) \boldsymbol{\mu})^{\alpha}|h(\boldsymbol{Z}(n))|^{\alpha} 1_{\{\boldsymbol{Z}(n) \neq \mathbf{0}\}}\right] \\
& +(\mathrm{E}[\boldsymbol{Z}(n+1) \boldsymbol{\mu} \mid \boldsymbol{Z}(n)=\mathbf{0}])^{\alpha} \\
\leq & \mathrm{E}\left[(\boldsymbol{Z}(n) \boldsymbol{\mu})^{\alpha} \hat{h}^{\alpha}(\boldsymbol{Z}(n) \mathbf{1})\right]+C_{1} \\
\leq & C_{2} \mathrm{E}\left[(\boldsymbol{Z}(n) \mathbf{1})^{\alpha}\right] \hat{h}^{\alpha}\left(\|\boldsymbol{Z}(n) \mathbf{1}\|_{\alpha}\right)+C_{1} .
\end{aligned}
$$

Furthermore, by applying Lemma A.2 to $\left\{n^{-1} \bar{g}_{\alpha}(n)\right\}_{n \geq 1}$, we find that there exists a function $\hat{g}_{\alpha}(x)$ such that $\bar{g}_{\alpha}(n) \leq \hat{g}_{\alpha}(n), x^{-1} \hat{g}_{\alpha}(x)$ is nonincreasing, $\int_{1}^{\infty} x^{-2} \hat{g}_{\alpha}(x) \mathrm{d} x<\infty$, and $\hat{g}_{\alpha}^{\alpha}\left(x^{1 / \alpha}\right)$ is concave. From Jensen's inequality, it follows that

$$
\begin{aligned}
\mathrm{E}\left[|\boldsymbol{Z}(n+1) \boldsymbol{\mu}-\mathrm{E}[\boldsymbol{Z}(n+1) \boldsymbol{\mu} \mid \boldsymbol{Z}(n)]|^{\alpha}\right] & =\mathrm{E}\left[G_{\alpha}^{\alpha}(\boldsymbol{Z}(n))\right] \\
& \leq \mathrm{E}\left[\hat{g}_{\alpha}^{\alpha}(\boldsymbol{Z}(n) \mathbf{1})\right] \\
& \leq \hat{g}_{\alpha}^{\alpha}\left(\|\boldsymbol{Z}(n) \mathbf{1}\|_{\alpha}\right) .
\end{aligned}
$$

Since $\mathrm{P}[\boldsymbol{Z}(n) \boldsymbol{\mu} \rightarrow \infty]>0$, there exists an $n_{0} \geq 0$ such that $\|W(n)\|_{\alpha}>0$ for all $n \geq n_{0}$. Moreover, since $0<\lim _{n \rightarrow \infty} \mathrm{E}[W(n)]<\infty$ (see Theorem 2.1), from (2.11), (2.12), and (2.13), we have

$$
\begin{aligned}
\|W(n+1)-W(n)\|_{\alpha} & \leq \frac{\|\boldsymbol{Z}(n) \mathbf{1}\|_{\alpha}}{r^{n+1}}\left(\frac{\hat{g}_{\alpha}\left(\|\boldsymbol{Z}(n) \mathbf{1}\|_{\alpha}\right)+C_{1}^{1 / \alpha}}{\|\boldsymbol{Z}(n) \mathbf{1}\|_{\alpha}}+C_{2}^{1 / \alpha} \hat{h}\left(\|\boldsymbol{Z}(n) \mathbf{1}\|_{\alpha}\right)\right) \\
& \leq C_{3} f\left(\|\boldsymbol{Z}(n) \mathbf{1}\|_{\alpha}\right)
\end{aligned}
$$

for all $n \geq n_{0}$, with $f(x):=x^{-1}\left(\hat{g}_{\alpha}(x)+C_{1}^{1 / \alpha}\right)+C_{2}^{1 / \alpha} \hat{h}(x)$ and $C_{3}>0$. Moreover, since $f(x)$ is nonincreasing and there exists a constant $\delta>0$ such that $\|\boldsymbol{Z}(n) \boldsymbol{\mu}\|_{\alpha} \geq\|\boldsymbol{Z}(n) \boldsymbol{\mu}\|_{1} \geq \delta r^{n}$ for every $n \geq n_{0}$, we then have

$$
\|W(n+1)-W(n)\|_{\alpha} \leq C_{3} f\left(C_{4} \delta r^{n}\right),
$$

for some constant $C_{4}>0$. Therefore, $r>1$ and $\int_{1}^{\infty} x^{-1} f(x) \mathrm{d} x<\infty$ imply that $\sum_{n=1}^{\infty} f\left(C_{4} \delta r^{n}\right)<\infty$, and, consequently, the sequence $\{W(n)\}_{n \geq 0}$ satisfies the $L^{\alpha}$-Cauchy convergence criterion. This completes the proof.

Remark 2.1. It is possible to obtain another set of sufficient conditions, equivalent to the previous one, from the function $\widetilde{G}_{\alpha}(z):=\left(\mathrm{E}\left[|\boldsymbol{Z}(n+1) \boldsymbol{\mu}-r \boldsymbol{Z}(n) \boldsymbol{\mu}|^{\alpha} \mid \boldsymbol{Z}(n)=\boldsymbol{z}\right]\right)^{1 / \alpha}$.

Remark 2.2. The conditions on the previous results are satisfied if, for example, $\bar{h}(n)=$ $O\left(\log ^{-\beta_{1}} n\right)$ and $\bar{g}_{\alpha}(n)=O\left(n \log ^{-\beta_{2}} n\right)$ for some constants $\beta_{1}, \beta_{2}>1$. Furthermore, notice that these conditions are given in terms of the norm one. Since all the norms on $\mathbb{R}^{m}$ are equivalent, these conditions can be expressed in terms of an arbitrary norm $\|\cdot\|$ in the following manner, where $\left\{x_{n}\right\}_{n \geq 1}=\left\{x \in \mathbb{R}_{+}: x=\|z\|, z \neq \mathbf{0}, z \in \mathbb{N}_{0}^{m}\right\}=: \mathbb{R}_{\|\cdot\|}$, such that $x_{n}<x_{n+1}$ for all $n \geq 1$ :

(i) $|h(z)| \leq \bar{h}(\|z\|)$ for all non-null vectors $z, \bar{h}\left(x_{n}\right) \geq \bar{h}\left(x_{n+1}\right)$ for all $n \geq 1$, and

$$
\sum_{n=1}^{\infty} x_{n}^{-1}\left(x_{n+1}-x_{n}\right) \bar{h}\left(x_{n}\right)<\infty .
$$


(ii) $G_{\alpha}(z) \leq \bar{g}_{\alpha}(\|z\|)$ for all non-null vectors $z, x_{n}^{-1} \bar{g}_{\alpha}\left(x_{n}\right) \geq x_{n+1}^{-1} \bar{g}_{\alpha}\left(x_{n+1}\right)$ for all $n \geq 1$, and

$$
\sum_{n=1}^{\infty} x_{n}^{-2}\left(x_{n+1}-x_{n}\right) \bar{g}_{\alpha}\left(x_{n}\right)<\infty \text {. }
$$

\section{Asymptotic behaviour of $\{Z(n)\}_{n \geq 0}$}

In the previous section, for each $\mu \in \mathbb{R}_{+}^{m}$ satisfying (2.1), and under some conditions, we proved that the sequence $\left\{r^{-n} \boldsymbol{Z}(n) \boldsymbol{\mu}\right\}_{n \geq 0}$ converges to a random variable nondegenerate at 0 . As a consequence, we can now prove the almost-sure convergence and $L^{\alpha}$-convergence $(1 \leq \alpha \leq 2)$ of $\left\{r^{-n} \boldsymbol{Z}(n)\right\}_{n \geq 0}$ to a random vector $\tilde{\boldsymbol{W}}$, concentrated in a one-dimensional subspace of $\mathbb{R}^{m}$, whose magnitude is given by the limit of a sequence $\left\{r^{-n} \boldsymbol{Z}(n) \boldsymbol{\mu}\right\}_{n \geq 0}$ for a particular $\boldsymbol{\mu} \in \mathbb{R}_{+}^{m}$.

We need to introduce new notation and assumptions. Let us impose the following condition on the transition vector of means of the chain (throughout, in products vectors are taken to be row or column vectors as appropriate):

$$
\mathrm{E}[\boldsymbol{Z}(n+1) \mid \boldsymbol{Z}(n)=\boldsymbol{z}]=\boldsymbol{z} \tilde{\boldsymbol{M}}+\tilde{\boldsymbol{h}}(\boldsymbol{z}), \quad \boldsymbol{z} \in \mathbb{N}_{0}^{m} .
$$

Here, $\tilde{\boldsymbol{M}}$ is a matrix of order $m$ with nonnegative coefficients and $\tilde{\boldsymbol{h}}(\boldsymbol{z})$ is a function from $\mathbb{R}^{m}$ to $\mathbb{R}^{m}$ such that $\tilde{h}_{j}(\boldsymbol{z})=o(\|z\|)$ for all $j \in\{1, \ldots, m\}$. We also suppose that the matrix $\tilde{\boldsymbol{M}}$ is positively regular. Therefore, if we denote by $\tilde{\rho}$ its Perron-Frobenius eigenvalue and by $\tilde{\boldsymbol{\mu}} \in \mathbb{R}_{+}^{m}$ one of its associated right eigenvectors (see [6]), it follows that

$$
\lim _{\|z\| \rightarrow \infty} \frac{\mathrm{E}[\boldsymbol{Z}(n+1) \tilde{\boldsymbol{\mu}} \mid \boldsymbol{Z}(n)=\boldsymbol{z}]}{\boldsymbol{z} \tilde{\boldsymbol{\mu}}}=\tilde{\rho},
$$

and, consequently, (2.1) is equivalent to $\tilde{\rho}>1$.

Let $\tilde{\boldsymbol{\mu}}^{(1)}, \ldots, \tilde{\boldsymbol{\mu}}^{(m)}$ be a basis of right eigenvectors and right generalized eigenvectors of $\tilde{\boldsymbol{M}}$, such that $\tilde{\boldsymbol{\mu}}^{(1)}=\tilde{\boldsymbol{\mu}}$. Let $\tilde{\boldsymbol{v}} \in \mathbb{R}_{+}^{m}$ be the left eigenvector associated to $\tilde{\rho}$ satisfying $\tilde{\boldsymbol{v}} \tilde{\boldsymbol{\mu}}=1$ and, consequently, $\tilde{\boldsymbol{v}} \tilde{\boldsymbol{\mu}}^{(i)}=0$ for each $i \in\{2, \ldots, m\}$. Finally we define $G_{\alpha}^{(i)}(z)$, for each $z \in \mathbb{N}_{0}^{m}$, $i \in\{1, \ldots, m\}$, and $\alpha, 1 \leq \alpha \leq 2$, by

$$
G_{\alpha}^{(i)}(z):=\left(\mathrm{E}\left[\left|\boldsymbol{Z}(n+1) \tilde{\boldsymbol{\mu}}^{(i)}-\mathrm{E}\left[\boldsymbol{Z}(n+1) \tilde{\boldsymbol{\mu}}^{(i)} \mid \boldsymbol{Z}(n)=\boldsymbol{z}\right]\right|^{\alpha} \mid \boldsymbol{Z}(n)=\boldsymbol{z}\right]\right)^{1 / \alpha} .
$$

Note that $G_{\alpha}^{(1)}(z)$ is equal to $G_{\tilde{\mu}, \alpha}(z)$. We can now formulate the following result.

Theorem 3.1. Let $\{\boldsymbol{Z}(n)\}_{n \geq 0}$ be an HMMC satisfying (3.1) with $\tilde{\rho}>1$ and such that $\mathrm{P}[\|\boldsymbol{Z}(n)\| \rightarrow \infty]>0$. Also let $1 \leq \alpha \leq 2$ and suppose that, for each $i \in\{1, \ldots, m\}$, there exist sequences $\left\{\bar{h}^{(i)}(n)\right\}_{n \geq 1}$ and $\left\{\bar{g}_{\alpha}^{(i)}(n)\right\}_{n \geq 1}$ such that

(i) for each non-null vector $z$ and $i \in\{1, \ldots, m\}$,

$$
\left|\tilde{\boldsymbol{h}}(\boldsymbol{z}) \tilde{\boldsymbol{\mu}}^{(i)}\right| \leq(\boldsymbol{z} \mathbf{1}) \bar{h}^{(i)}(\boldsymbol{z} \mathbf{1}) \text { and } G_{\alpha}^{(i)}(z) \leq \bar{g}_{\alpha}^{(i)}(\boldsymbol{z} \mathbf{1}),
$$

and

(ii) $\left\{\bar{h}^{(i)}(n)\right\}_{n \geq 1}$ and $\left\{n^{-1} \bar{g}_{\alpha}^{(i)}(n)\right\}_{n \geq 1}$ are nonincreasing and

$$
\sum_{n=1}^{\infty} n^{-1} \bar{h}^{(i)}(n)<\infty, \quad \sum_{n=1}^{\infty} n^{-2} \bar{g}_{\alpha}^{(i)}(n)<\infty .
$$


Then the sequence $\left\{\tilde{\rho}^{-n} \boldsymbol{Z}(n)\right\}_{n \geq 0}$ converges almost surely and in $L^{\alpha}$ to a finite and random vector, $\tilde{W}:=\tilde{\mathbf{v}} W$, nondegenerate at $\mathbf{0}$, where $W$ is the almost-sure limit of the sequence $\left\{\tilde{\rho}^{-n} \boldsymbol{Z}(n) \tilde{\boldsymbol{\mu}}\right\}_{n \geq 0}$.

Proof. In order to prove the result, we apply a similar reasoning to that used in [4] in the context of population-size-dependent multitype branching processes.

First we observe that if $\left\{\tilde{\rho}^{-n} \boldsymbol{Z}(n)\right\}_{n \geq 0}$ converges in $L^{\alpha}$ to a limit, $\tilde{\boldsymbol{W}}$, then this limit will be nondegenerate at $\mathbf{0}$ if and only if the limit of the process $\left\{\tilde{\rho}^{-n} \boldsymbol{Z}(n) \tilde{\boldsymbol{\mu}}^{(1)}\right\}_{n \geq 0}$ is nondegenerate at 0 .

The growth rate of the process $\left\{\boldsymbol{Z}(n) \tilde{\boldsymbol{\mu}}^{(1)}\right\}_{n \geq 0}$ is $r=\tilde{\rho}$, and it follows that $h(z)=$ $\left(z \tilde{\boldsymbol{\mu}}^{(1)}\right)^{-1} \tilde{\boldsymbol{h}}(\boldsymbol{z}) \tilde{\boldsymbol{\mu}}^{(1)}$ for all non-null vectors $\boldsymbol{z}$. By taking into account the equivalence of the norms on $\mathbb{R}^{m}$, we see that $|h(z)|$ is bounded by $C_{1} \bar{h}^{(1)}(z \mathbf{1})$ for some constant $C_{1}>0$. Then, by applying Proposition 2.2 and Theorem 2.2 to such a process, with $\bar{h}(n)=C_{1} \bar{h}^{(1)}(n)$ and $\bar{g}_{\alpha}(n)=\bar{g}_{\alpha}^{(1)}(n)$, we find that

$$
\lim _{n \rightarrow \infty} \tilde{\rho}^{-n} \boldsymbol{Z}(n) \tilde{\boldsymbol{\mu}}^{(1)}=W \quad \text { a.s. and in } L^{\alpha},
$$

where $W$ is finite and nondegenerate at 0 .

To complete the proof it is sufficient to prove that, for each $i \in\{2, \ldots, m\}$,

$$
\lim _{n \rightarrow \infty} \tilde{\rho}^{-n} \boldsymbol{Z}(n) \tilde{\boldsymbol{\mu}}^{(i)}=0 \quad \text { a.s. and in } L^{\alpha} .
$$

Indeed, if the previous equality holds then, for each $\boldsymbol{a} \in \mathbb{R}^{m}$,

$$
\lim _{n \rightarrow \infty} \tilde{\rho}^{-n} \boldsymbol{Z}(n) \boldsymbol{a}=\lim _{n \rightarrow \infty} \sum_{i=1}^{m} a_{i} \tilde{\rho}^{-n} \boldsymbol{Z}(n) \tilde{\boldsymbol{\mu}}^{(i)}=a_{1} W \quad \text { a.s. and in } L^{\alpha},
$$

where $a_{1}, \ldots, a_{m}$ are the coordinates of $\boldsymbol{a}$ in the basis $\tilde{\boldsymbol{\mu}}^{(1)}, \ldots, \tilde{\boldsymbol{\mu}}^{(m)}$. Moreover, since $\tilde{\boldsymbol{v}} \tilde{\boldsymbol{\mu}}^{(i)}=$ 0 for $i \in\{2, \ldots, m\}$, we have $a_{1}=\tilde{\boldsymbol{v}} \boldsymbol{a}$. Therefore, for each $j \in\{1, \ldots, m\},\left\{\tilde{\rho}^{-n} Z_{j}(n)\right\}_{n \geq 0}$ converges almost surely and in $L^{\alpha}$ to $\tilde{v}_{j} W$, which implies the almost-sure convergence and $L^{\alpha}$-convergence of $\left\{\tilde{\rho}^{-n} \boldsymbol{Z}(n)\right\}_{n \geq 0}$ to $\tilde{\boldsymbol{v}} W$.

Thus, to finish the proof, let us show that, for each $i \in\{2, \ldots, m\}$,

$$
\sum_{n=0}^{\infty}\left\|\tilde{\rho}^{-n} \boldsymbol{Z}(n) \tilde{\boldsymbol{\mu}}^{(i)}\right\|_{\alpha}<\infty
$$

From (3.2) it immediately follows that $\tilde{\rho}^{-n} \boldsymbol{Z}(n) \tilde{\boldsymbol{\mu}}^{(i)}$ converges to 0 in $L^{\alpha}$ and, by applying Markov's inequality, we have, for every $\varepsilon>0$,

$$
\sum_{n=0}^{\infty} \mathrm{P}\left[\left|\tilde{\rho}^{-n} \boldsymbol{Z}(n) \tilde{\boldsymbol{\mu}}^{(i)}\right|>\varepsilon\right]<\infty .
$$

The complete, and, therefore, the almost-sure, convergence of the sequence $\left\{\tilde{\rho}^{-n} \boldsymbol{Z}(n) \tilde{\boldsymbol{\mu}}^{(i)}\right\}_{n \geq 0}$ to 0 follows.

Since $\tilde{\boldsymbol{M}}$ is positively regular, the eigenvalue $\tilde{\rho}$ has multiplicity 1 and $|\lambda|<\tilde{\rho}$ for any other eigenvalue $\lambda$. Suppose that $\lambda$ is an eigenvalue with multiplicity $s \geq 1$, corresponding to the right generalized eigenvectors $\tilde{\boldsymbol{\mu}}^{\left(i_{1}\right)}, \ldots, \tilde{\boldsymbol{\mu}}^{\left(i_{s}\right)}$; that is,

$$
\tilde{\boldsymbol{M}} \tilde{\boldsymbol{\mu}}^{\left(i_{1}\right)}=\lambda \tilde{\boldsymbol{\mu}}^{\left(i_{1}\right)}, \quad \tilde{\boldsymbol{M}} \tilde{\boldsymbol{\mu}}^{\left(i_{t}\right)}=\lambda \tilde{\boldsymbol{\mu}}^{\left(i_{t}\right)}+\tilde{\boldsymbol{\mu}}^{\left(i_{t-1}\right)}, \quad t \in\{2, \ldots, s\} .
$$


Let us prove by induction on $t$ that, for each $t \in\{1, \ldots, s\}$,

$$
\sum_{n=0}^{\infty}\left\|\tilde{\rho}^{-n} \boldsymbol{Z}(n) \tilde{\boldsymbol{\mu}}^{\left(i_{t}\right)}\right\|_{\alpha}<\infty .
$$

For $t=1$, using (3.1) and a procedure similar to the one applied in deriving (2.12) and (2.13), we have

$$
\begin{aligned}
\left\|\boldsymbol{Z}(n+1) \tilde{\boldsymbol{\mu}}^{\left(i_{1}\right)}\right\|_{\alpha} \leq & \left\|\boldsymbol{Z}(n+1) \tilde{\boldsymbol{\mu}}^{\left(i_{1}\right)}-\mathrm{E}\left[\boldsymbol{Z}(n+1) \tilde{\boldsymbol{\mu}}^{\left(i_{1}\right)} \mid \boldsymbol{Z}(n)\right]\right\|_{\alpha} \\
& +\left\|\mathrm{E}\left[\boldsymbol{Z}(n+1) \tilde{\boldsymbol{\mu}}^{\left(i_{1}\right)} \mid \boldsymbol{Z}(n)\right]\right\|_{\alpha} \\
\leq & |\lambda|\left\|\boldsymbol{Z}(n) \tilde{\boldsymbol{\mu}}^{\left(i_{1}\right)}\right\|_{\alpha}+\left\|(\boldsymbol{Z}(n) \mathbf{1}) \bar{h}^{\left(i_{1}\right)}(\boldsymbol{Z}(n) \mathbf{1})\right\|_{\alpha} \\
& +\left|\tilde{\boldsymbol{h}}(\mathbf{0}) \tilde{\boldsymbol{\mu}}^{\left(i_{1}\right)}\right|+\left\|\bar{g}_{\alpha}^{\left(i_{1}\right)}(\boldsymbol{Z}(n) \mathbf{1})\right\|_{\alpha} \\
\leq & |\lambda|\left\|\boldsymbol{Z}(n) \tilde{\boldsymbol{\mu}}^{\left(i_{1}\right)}\right\|_{\alpha}+\|\boldsymbol{Z}(n) \mathbf{1}\|_{\alpha} \hat{h}^{\left(i_{1}\right)}\left(\|\boldsymbol{Z}(n) \mathbf{1}\|_{\alpha}\right) \\
& +C_{2}+\hat{g}_{\alpha}^{\left(i_{1}\right)}\left(\|\boldsymbol{Z}(n) \mathbf{1}\|_{\alpha}\right)
\end{aligned}
$$

for some constant $C_{2}>0$, with $\hat{h}^{\left(i_{1}\right)}(x)$ and $\hat{g}_{\alpha}^{\left(i_{1}\right)}(x)$ nonincreasing functions such that $\int_{1}^{\infty} x^{-1} \hat{h}^{\left(i_{1}\right)}(x) \mathrm{d} x<\infty$ and $\int_{1}^{\infty} x^{-2} \hat{g}_{\alpha}^{\left(i_{1}\right)}(x) \mathrm{d} x<\infty$. By setting

$$
f^{\left(i_{1}\right)}(x):=x^{-1}\left(\hat{g}_{\alpha}^{\left(i_{1}\right)}(x)+C_{2}\right)+\hat{h}^{\left(i_{1}\right)}(x),
$$

we find that $f^{\left(i_{1}\right)}(x)$ is nonincreasing and $\int_{1}^{\infty} x^{-1} f^{\left(i_{1}\right)}(x) \mathrm{d} x<\infty$.

Since $\mathrm{P}[\|\boldsymbol{Z}(n)\| \rightarrow \infty]>0$, there exists an $n_{0} \geq 0$ such that $\|\boldsymbol{Z}(n) \mathbf{1}\|_{\alpha}>0$ for all $n \geq n_{0}$. Therefore, from (3.3), we have

$$
\begin{aligned}
\left\|\tilde{\rho}^{-n-1} \boldsymbol{Z}(n+1) \tilde{\boldsymbol{\mu}}^{\left(i_{1}\right)}\right\|_{\alpha} \leq & \tilde{\rho}^{-1}|\lambda|\left\|\tilde{\rho}^{-n} \boldsymbol{Z}(n) \tilde{\boldsymbol{\mu}}^{\left(i_{1}\right)}\right\|_{\alpha} \\
& +\tilde{\rho}^{-1} f^{\left(i_{1}\right)}\left(\|\boldsymbol{Z}(n) \mathbf{1}\|_{\alpha}\right)\left\|\tilde{\rho}^{-n} \boldsymbol{Z}(n) \mathbf{1}\right\|_{\alpha}
\end{aligned}
$$

for all $n \geq n_{0}$. The sequence $\left\{\left\|\tilde{\rho}^{-n} \boldsymbol{Z}(n) \mathbf{1}\right\|_{\alpha}\right\}_{n \geq 0}$ is bounded because $\left\{\tilde{\rho}^{-n} \boldsymbol{Z}(n) \tilde{\boldsymbol{\mu}}^{(1)}\right\}_{n \geq 0}$ converges in $L^{\alpha}$ and all the norms on $\mathbb{R}^{m}$ are equivalent. Also, since the limit is nondegenerate at 0 , there exists a $\delta>0$ such that $\|\boldsymbol{Z}(n) \mathbf{1}\|_{\alpha} \geq\|\boldsymbol{Z}(n) \mathbf{1}\|_{1} \geq \delta \tilde{\rho}^{n}$ for every $n \geq n_{0}$, implying that

$$
\sum_{n=n_{0}}^{\infty} f^{\left(i_{1}\right)}\left(\|\boldsymbol{Z}(n) \mathbf{1}\|_{\alpha}\right) \leq \sum_{n=n_{0}}^{\infty} f^{\left(i_{1}\right)}\left(\delta \tilde{\rho}^{n}\right)<\infty,
$$

because $\int_{1}^{\infty} x^{-1} f^{\left(i_{1}\right)}(x) \mathrm{d} x<\infty$ and $\tilde{\rho}>1$. Therefore, by virtue of Lemma A.4, it follows that

$$
\sum_{n=n_{0}}^{\infty}\left\|\tilde{\rho}^{-n} \boldsymbol{Z}(n) \tilde{\boldsymbol{\mu}}^{\left(i_{1}\right)}\right\|_{\alpha}<\infty .
$$

To conclude, let us consider $t \in\{2, \ldots, s\}$. Then

$$
\begin{aligned}
\left\|\boldsymbol{Z}(n+1) \tilde{\boldsymbol{\mu}}^{\left(i_{t}\right)}\right\|_{\alpha} \leq & \left\|\boldsymbol{Z}(n+1) \tilde{\boldsymbol{\mu}}^{\left(i_{t}\right)}-\mathrm{E}\left[\boldsymbol{Z}(n+1) \tilde{\boldsymbol{\mu}}^{\left(i_{t}\right)} \mid \boldsymbol{Z}(n)\right]\right\|_{\alpha} \\
& +\left\|\mathrm{E}\left[\boldsymbol{Z}(n+1) \tilde{\boldsymbol{\mu}}^{\left(i_{t}\right)} \mid \boldsymbol{Z}(n)\right]\right\|_{\alpha} \\
\leq & |\lambda|\left\|\boldsymbol{Z}(n) \tilde{\boldsymbol{\mu}}^{\left(i_{t}\right)}\right\|_{\alpha}+\left\|\boldsymbol{Z}(n) \tilde{\boldsymbol{\mu}}^{\left(i_{t-1}\right)}\right\|_{\alpha}+\left|\tilde{\boldsymbol{h}}(\mathbf{0}) \tilde{\boldsymbol{\mu}}^{\left(i_{t}\right)}\right| \\
& +\left\|(\boldsymbol{Z}(n) \mathbf{1}) \bar{h}^{\left(i_{t}\right)}(\boldsymbol{Z}(n) \mathbf{1})\right\|_{\alpha}+\left\|\bar{g}_{\alpha}^{\left(i_{t}\right)}(\boldsymbol{Z}(n) \mathbf{1})\right\|_{\alpha} .
\end{aligned}
$$


By the induction hypothesis, $\sum_{n=0}^{\infty}\left\|\tilde{\rho}^{-n} \boldsymbol{Z}(n) \tilde{\boldsymbol{\mu}}^{\left(i_{t-1}\right)}\right\|_{\alpha}<\infty$ and, using arguments similar to those used in the case $t=1$, we have

$$
\sum_{n=0}^{\infty} \tilde{\rho}^{-n}\left[\left\|(\boldsymbol{Z}(n) \mathbf{1}) \bar{h}^{\left(i_{t}\right)}(\boldsymbol{Z}(n) \mathbf{1})\right\|_{\alpha}+\left|\tilde{\boldsymbol{h}}(\mathbf{0}) \tilde{\boldsymbol{\mu}}^{\left(i_{t}\right)}\right|+\left\|\bar{g}_{\alpha}^{\left(i_{t}\right)}(\boldsymbol{Z}(n) \mathbf{1})\right\|_{\alpha}\right]<\infty
$$

Applying Lemma A.4 once more, we conclude that

$$
\sum_{n=0}^{\infty}\left\|\tilde{\rho}^{-n} \boldsymbol{Z}(n) \tilde{\boldsymbol{\mu}}^{\left(i_{t}\right)}\right\|_{\alpha}<\infty
$$

completing the proof.

Remark 3.1. Notice that although the random variable $W$ depends on the right eigenvector $\tilde{\boldsymbol{\mu}}$ chosen, the random vector $\tilde{\boldsymbol{W}}=\tilde{\boldsymbol{v}} W$ does not.

Remark 3.2. Under the conditions of the previous theorem, an HMMC exhibits the same behaviour as a supercritical, positively regular multitype branching process (see [5]); that is, $\tilde{\boldsymbol{W}}$ has a fixed direction given by $\tilde{\boldsymbol{v}}$ and a random magnitude provided by the limit of the sequence $\left\{\tilde{\rho}^{-n} \boldsymbol{Z}(n) \tilde{\boldsymbol{\mu}}\right\}_{n \geq 0}$. Moreover, since $\tilde{\boldsymbol{v}} \in \mathbb{R}_{+}^{m}$ and $\mathrm{P}[W>0]>0$ we deduce that

$$
\mathrm{P}\left[Z_{i}(n) \rightarrow \infty, 1 \leq i \leq m\right]>0 .
$$

\section{On controlled multitype branching processes}

In this section, we apply the results obtained for HMMCs to a class of controlled multitype branching process introduced in [1], where the production of new individuals depends on the size of the population. This model generalizes both the controlled multitype branching process, considered in [7], and the population-size-dependent multitype branching process, studied in [4]. In addition, randomness in the control function and dependence between the individuals in the same generation at the reproduction time are allowed. These properties make the model more accurate in describing some real situations. Mathematically, we consider a sequence of $m$-dimensional random vectors $\{\boldsymbol{Z}(n)\}_{n \geq 0}$ defined recursively by

$$
\boldsymbol{Z}(0)=\boldsymbol{z}^{(0)} \in \mathbb{N}_{0}^{m}, \quad \boldsymbol{Z}(n+1)=\sum_{i=1}^{m} \sum_{j=1}^{\phi_{i}^{n}(\boldsymbol{Z}(n))} \boldsymbol{X}^{i, n, j}(\boldsymbol{Z}(n)), \quad n \geq 0 .
$$

Here, $\left\{\boldsymbol{\phi}^{n}(\boldsymbol{z}), n=0,1, \ldots, \boldsymbol{z} \in \mathbb{N}_{0}^{m}\right\}$ and

$$
\left\{\boldsymbol{X}^{i, n, j}(\boldsymbol{z}), i=1, \ldots, m, n=0,1, \ldots, j=1,2, \ldots, \boldsymbol{z} \in \mathbb{N}_{0}^{m}\right\}
$$

are independent sequences of $m$-dimensional, nonnegative, integer-component random vectors defined on the same probability triple $(\Omega, \mathcal{A}, \mathrm{P})$. They have the following properties.

(i) The stochastic processes $\left\{\boldsymbol{\phi}^{n}(\boldsymbol{z}), \boldsymbol{z} \in \mathbb{N}_{0}^{m}\right\}, n \geq 0$, are independent and, for each $z \in \mathbb{N}_{0}^{m}$, the vectors $\left\{\boldsymbol{\phi}^{n}(\boldsymbol{z}), n=0,1, \ldots\right\}$ are identically distributed.

(ii) The stochastic processes $\left\{\boldsymbol{X}^{i, n, j}(\boldsymbol{z}), i=1, \ldots, m, j=1,2, \ldots, \boldsymbol{z} \in \mathbb{N}_{0}^{m}\right\}, n \geq 0$, are independent and identically distributed. Moreover, for each $i \in\{1, \ldots, m\}$ and $z \in \mathbb{N}_{0}^{m}$, the vectors $\left\{\boldsymbol{X}^{i, n, j}(\boldsymbol{z}), n=0,1, \ldots, j=1,2, \ldots\right\}$ are identically distributed. 
The stochastic process $\{\boldsymbol{Z}(n)\}_{n \geq 0}$ is called a controlled multitype branching process with random control and population-size-dependent reproduction (CMPD).

From the definition, it follows that a CMPD is an HMMC. Moreover, taking into account the independence between reproduction and control, it follows that, for each $z \in \mathbb{N}_{0}^{m}$,

$$
\mathrm{E}[\boldsymbol{Z}(n+1) \mid \boldsymbol{Z}(n)=\boldsymbol{z}]=\sum_{i=1}^{m} \mathrm{E}\left[\phi_{i}^{0}(\boldsymbol{z})\right] \mathrm{E}\left[\boldsymbol{X}^{i, 0,1}(\boldsymbol{z})\right]
$$

It seems natural that the conditions we are searching for can be written in terms of $\mathrm{E}\left[\phi_{i}^{0}(\boldsymbol{z})\right]$ and $\mathrm{E}\left[\boldsymbol{X}^{i, 0,1}(\boldsymbol{z})\right]$. More specifically, we will make the following assumptions.

Assumption 4.1. There exist $m_{i j} \geq 0, i, j \in\{1, \ldots, m\}$, such that

$$
\mathrm{E}\left[X_{j}^{i, 0,1}(z)\right]=m_{i j}+h_{i j}^{\mathrm{R}}(z), \quad \text { where } h_{i j}^{\mathrm{R}}: \mathbb{R}^{m} \rightarrow \mathbb{R}, h_{i j}^{\mathrm{R}}(z)=o(1) .
$$

Assumption 4.2. There exist $\lambda_{i} \geq 0, i \in\{1, \ldots, m\}$, such that

$$
\mathrm{E}\left[\phi_{i}^{0}(z)\right]=\lambda_{i} z_{i}+h_{i}^{\mathrm{c}}(z), \text { where } h_{i}^{\mathrm{c}}: \mathbb{R}^{m} \rightarrow \mathbb{R}, h_{i}^{\mathrm{c}}(z)=o(\|z\|) .
$$

Assumption 4.1 is a hypothesis commonly used in population-size-dependent models; a possible intuitive interpretation of its effect is the stabilization of the average number of descendants per individual as the population size grows indefinitely.

On the other hand, Assumption 4.2 implies that the average number of progenitors of a certain type is proportional to the number of individuals of that type plus or minus some number of progenitors that is negligible with respect to the total population. Notice that, under Assumption 4.2, immigration or emigration of progenitors of each type is allowed. Immigration is possible even if there are no individuals of a given type. This could not happen if $h_{i}^{\mathrm{c}}(z)=z_{i} o(1)$. However, in this case we could determine $\lambda_{i}$ explicitly according to $\lambda_{i}=\lim _{\|z\| \rightarrow \infty, z_{i} \neq 0} z_{i}^{-1} \mathrm{E}\left[\phi_{i}^{0}(z)\right]$.

Given Assumptions 4.1 and 4.2, (3.1) holds with $\tilde{\boldsymbol{M}}:=\left(\lambda_{i} m_{i j}\right)_{1 \leq i, j \leq m}$ and

$$
\tilde{h}_{j}(z)=\sum_{i=1}^{m}\left(\lambda_{i} z_{i} h_{i j}^{\mathrm{R}}(z)+h_{i}^{\mathrm{c}}(z) m_{i j}+h_{i}^{\mathrm{c}}(z) h_{i j}^{\mathrm{R}}(z)\right)
$$

for each $j \in\{1, \ldots, m\}$. Moreover, the matrix $\left(\lambda_{i} m_{i j}\right)_{1 \leq i, j \leq m}$ is positively regular if and only if $\lambda_{i}>0$ for all $i \in\{1, \ldots, m\}$ and the matrix $\left(m_{i j}\right)_{1 \leq i, j \leq m}$ is positively regular.

In order to apply the results proved in the previous sections, let us conveniently bound $\left|\tilde{\boldsymbol{h}}(\boldsymbol{z}) \tilde{\boldsymbol{\mu}}^{(i)}\right|$ and $G_{\alpha}^{(i)}(\boldsymbol{z})$, where $z \in \mathbb{N}_{0}^{m}, i \in\{1, \ldots, m\}$, and $1 \leq \alpha \leq 2$. Since Assumption 4.1 holds, it follows that

$$
\begin{aligned}
\left|\tilde{\boldsymbol{h}}(\boldsymbol{z}) \tilde{\boldsymbol{\mu}}^{(i)}\right| & =\left|\sum_{j=1}^{m}\left(\sum_{k=1}^{m}\left(\lambda_{k} z_{k} h_{k j}^{\mathrm{R}}(\boldsymbol{z})+h_{k}^{\mathrm{c}}(\boldsymbol{z}) m_{k j}+h_{k}^{\mathrm{c}}(\boldsymbol{z}) h_{k j}^{\mathrm{R}}(\boldsymbol{z})\right)\right) \tilde{\mu}_{j}^{(i)}\right| \\
& \leq C_{1}(\boldsymbol{z} \mathbf{1}) \max _{1 \leq k, j \leq m}\left\{\left|h_{k j}^{\mathrm{R}}(\boldsymbol{z})\right|\right\}+C_{2} \max _{1 \leq k \leq m}\left\{\left|h_{k}^{\mathrm{c}}(\boldsymbol{z})\right|\right\}
\end{aligned}
$$


for some constants $C_{1}, C_{2}>0$, and

$$
\begin{aligned}
G_{\alpha}^{(i)}(\boldsymbol{z})= & \left(\mathrm{E}\left[\left|\boldsymbol{Z}(n+1) \tilde{\boldsymbol{\mu}}^{(i)}-\mathrm{E}\left[\boldsymbol{Z}(n+1) \tilde{\boldsymbol{\mu}}^{(i)} \mid \boldsymbol{Z}(n)=\boldsymbol{z}\right]\right|^{\alpha} \mid \boldsymbol{Z}(n)=\boldsymbol{z}\right]\right)^{1 / \alpha} \\
= & \left\|\sum_{l=1}^{m} \sum_{j=1}^{m}\left(\sum_{k=1}^{\phi_{l}^{0}(\boldsymbol{z})} X_{j}^{l, 0, k}(\boldsymbol{z})-\mathrm{E}\left[\phi_{l}^{0}(\boldsymbol{z})\right] \mathrm{E}\left[X_{j}^{l, 0,1}(\boldsymbol{z})\right]\right) \tilde{\mu}_{j}^{(i)}\right\|_{\alpha} \\
\leq & \sum_{l=1}^{m} \sum_{j=1}^{m}\left|\tilde{\mu}_{j}^{(i)}\right|\left\|\sum_{k=1}^{\phi_{l}^{0}(z)}\left(X_{j}^{l, 0, k}(\boldsymbol{z})-\mathrm{E}\left[X_{j}^{l, 0,1}(\boldsymbol{z})\right]\right)\right\|_{\alpha} \\
& +\sum_{l=1}^{m} \sum_{j=1}^{m}\left|\tilde{\mu}_{j}^{(i)}\right| \mathrm{E}\left[X_{j}^{l, 0,1}(\boldsymbol{z})\right]\left\|\phi_{l}^{0}(z)-\mathrm{E}\left[\phi_{l}^{0}(\boldsymbol{z})\right]\right\|_{\alpha} .
\end{aligned}
$$

Furthermore,

$$
\left\|\sum_{k=1}^{\phi_{l}^{0}(z)}\left(X_{j}^{l, 0, k}(z)-\mathrm{E}\left[X_{j}^{l, 0,1}(z)\right]\right)\right\|_{\alpha} \leq\left\|\phi_{l}^{0}(z)\right\|_{\alpha}\left\|X_{j}^{l, 0,1}(z)-\mathrm{E}\left[X_{j}^{l, 0,1}(z)\right]\right\|_{\alpha} .
$$

On the other hand, if the random vectors $\boldsymbol{X}^{i, 0, k}(\boldsymbol{z}), k \geq 1, i \in\{1, \ldots, m\}$, are independent for each fixed $z \in \mathbb{N}_{0}^{m}$, then, since $1 \leq \alpha \leq 2$, and using the von Bahr-Esseen inequality (see [8]), we can establish the following bound:

$$
\left\|\sum_{k=1}^{\phi_{l}^{0}(z)}\left(X_{j}^{l, 0, k}(z)-\mathrm{E}\left[X_{j}^{l, 0,1}(z)\right]\right)\right\|_{\alpha} \leq \mathrm{E}\left[\phi_{l}^{0}(z)\right]^{1 / \alpha}\left\|X_{j}^{l, 0,1}(z)-\mathrm{E}\left[X_{j}^{l, 0,1}(z)\right]\right\|_{\alpha} .
$$

In [1], it was proved that $\mathrm{P}\left[\|\boldsymbol{Z}(n)\| \rightarrow \infty \mid \boldsymbol{Z}(0)=\boldsymbol{z}^{(0)}\right]>0$ if $\tilde{\rho}>1$, if $\boldsymbol{z}^{(0)}$ is large enough, and if, for some $\delta \geq 0$ and every $i, j \in\{1, \ldots, m\}$,

$$
\left\|\phi_{i}^{0}(z)-\mathrm{E}\left[\phi_{i}^{0}(z)\right]\right\|_{1+\delta}=O\left(\|z\|^{\delta /(1+\delta)}\right)
$$

and

$$
\left\|\phi_{i}^{0}(z)\right\|_{1+\delta}\left\|X_{j}^{i, 0,1}(z)-\mathrm{E}\left[X_{j}^{i, 0,1}(z)\right]\right\|_{1+\delta}=O\left(\|z\|^{\delta /(1+\delta)}\right) .
$$

In summary, we establish the following result for CMPDs.

Corollary 4.1. Let $\{\boldsymbol{Z}(n)\}_{n \geq 0}$ be a CMPD satisfying Assumptions 4.1 and 4.2, where the matrix $\left(\lambda_{i} m_{i j}\right)_{1 \leq i, j \leq m}$ is positively regular with Perron-Frobenius eigenvalue $\tilde{\rho}>1$. Also let $1 \leq \alpha \leq 2$ and suppose that there exist sequences $\left\{\bar{h}^{\mathrm{R}}(n)\right\}_{n \geq 1},\left\{\bar{h}^{\mathrm{c}}(n)\right\}_{n \geq 1}$, and $\left\{\bar{g}_{\alpha}(n)\right\}_{n \geq 1}$ such that

(i) for each non-null vector $z$,

$$
\begin{gathered}
\max _{1 \leq i, j \leq m}\left\{\left|h_{i j}^{\mathrm{R}}(\boldsymbol{z})\right|\right\} \leq \bar{h}^{\mathrm{R}}(\boldsymbol{z} \mathbf{1}), \quad \max _{1 \leq i \leq m}\left\{\left|h_{i}^{\mathrm{c}}(\boldsymbol{z})\right|\right\} \leq(\boldsymbol{z} \mathbf{1}) \bar{h}^{\mathrm{c}}(\boldsymbol{z} \mathbf{1}), \\
\max _{1 \leq i, j \leq m}\left\{\left\|\phi_{i}^{0}(\boldsymbol{z})-\mathrm{E}\left[\phi_{i}^{0}(\boldsymbol{z})\right]\right\|_{\alpha},\left\|\phi_{i}^{0}(\boldsymbol{z})\right\|_{\alpha}\left\|X_{j}^{i, 0,1}(\boldsymbol{z})-\mathrm{E}\left[X_{j}^{i, 0,1}(\boldsymbol{z})\right]\right\|_{\alpha}\right\} \leq \bar{g}_{\alpha}(\boldsymbol{z} \mathbf{1}) ;
\end{gathered}
$$


and

(ii) $\left\{\bar{h}^{\mathrm{R}}(n)\right\}_{n \geq 1},\left\{\bar{h}^{\mathrm{c}}(n)\right\}_{n \geq 1}$, and $\left\{n^{-1} \bar{g}_{\alpha}(n)\right\}_{n \geq 1}$ are nonincreasing and

$$
\sum_{n=1}^{\infty} n^{-1} \bar{h}^{\mathrm{R}}(n)<\infty, \quad \sum_{n=1}^{\infty} n^{-1} \bar{h}^{\mathrm{c}}(n)<\infty, \quad \sum_{n=1}^{\infty} n^{-2} \bar{g}_{\alpha}(n)<\infty .
$$

If (4.1) and (4.2) hold then the sequence $\left\{\tilde{\rho}^{-n} \boldsymbol{Z}(n)\right\}_{n \geq 0}$ converges almost surely and in $L^{\alpha}$ to a finite random vector, $\tilde{\boldsymbol{W}}$, nondegenerate at $\mathbf{0}$.

Remark 4.1. Under the assumption of independence of the reproduction vectors (the $X \mathrm{~s}$ ), (4.3) can be replaced by

$$
\max _{1 \leq i, j \leq m}\left\{\left\|\phi_{i}^{0}(z)-\mathrm{E}\left[\phi_{i}^{0}(z)\right]\right\|_{\alpha}, \mathrm{E}\left[\phi_{i}^{0}(z)\right]^{1 / \alpha}\left\|X_{j}^{i, 0, k}(z)-\mathrm{E}\left[X_{j}^{i, 0,1}(z)\right]\right\|_{\alpha}\right\} \leq \bar{g}_{\alpha}(\boldsymbol{z} \mathbf{1}) .
$$

Remark 4.2. The present study applies to the controlled multitype branching processes proposed in [7], which have not as yet been investigated. Also, the previous result extends those of [4] on the geometric growth of population-size-dependent multitype branching processes.

\section{Appendix A. Lemmas}

Lemma A.1. Let $\left\{a_{n}\right\}_{n \geq 1}$ be a nonincreasing sequence of positive real numbers. Then the function

$$
h(x):=\left(a_{1}+\int_{1}^{x^{1 / \alpha}} a_{\lfloor t\rfloor} \mathrm{d} t\right)^{\alpha},
$$

where $\lfloor t\rfloor$ denotes the greatest integer less than or equal to $t$, is concave on $x>1$ for any $\alpha$, $1 \leq \alpha \leq 2$.

Proof. From the definition of the function $h(x)$, it follows that

$$
h(x)= \begin{cases}x a_{1}^{\alpha} & \text { if } 1<x<2^{\alpha}, \\ \left(a_{1}+\sum_{k=1}^{n-1} a_{k}+a_{n}\left(x^{1 / \alpha}-n\right)\right)^{\alpha} & \text { if } n^{\alpha} \leq x<(n+1)^{\alpha}, n \geq 2 .\end{cases}
$$

Obviously $h(x)$ is a concave function for $x$ with $1<x<2^{\alpha}$. For $x$ with $n^{\alpha} \leq x<$ $(n+1)^{\alpha}, n \geq 2$, the function

$$
h^{\prime \prime}(x)=\frac{\alpha^{-1}(\alpha-1) a_{n} x^{(1-2 \alpha) / \alpha}}{\left(a_{1}+\sum_{k=1}^{n-1} a_{k}+a_{n}\left(x^{1 / \alpha}-n\right)\right)^{1-\alpha}}\left(\frac{a_{n} x^{1 / \alpha}}{a_{1}+\sum_{k=1}^{n-1} a_{k}+a_{n}\left(x^{1 / \alpha}-n\right)}-1\right)
$$

is nonpositive, because $\left\{a_{n}\right\}_{n \geq 1}$ is a nonincreasing sequence of positive real numbers and $n a_{n} \leq a_{1}+\sum_{k=1}^{n-1} a_{k}$. To conclude, let us prove that $h_{-}^{\prime}\left(n^{\alpha}\right) \geq h_{+}^{\prime}\left(n^{\alpha}\right), n \geq 2$, where $h_{-}^{\prime}\left(x_{0}\right)$ and $h_{+}^{\prime}\left(x_{0}\right)$ respectively denote the left-hand and the right-hand derivatives of $h(x)$ evaluated at $x_{0}$. Indeed,

$$
h_{-}^{\prime}\left(n^{\alpha}\right)=a_{n-1}\left(a_{1}+\sum_{k=1}^{n-1} a_{k}\right)^{\alpha-1} n^{1-\alpha} \geq a_{n}\left(a_{1}+\sum_{k=1}^{n-1} a_{k}\right)^{\alpha-1} n^{1-\alpha}=h_{+}^{\prime}\left(n^{\alpha}\right) .
$$


Lemma A.2. Let $\left\{a_{n}\right\}_{n \geq 0}$ be a nonincreasing sequence of positive real numbers such that $\sum_{n=1}^{\infty} n^{-1} a_{n}<\infty$. Then there exists a nonincreasing, positive function $a(x)$, defined on $\mathbb{R}_{+}$, such that $a_{n} \leq a(n)$ for every $n, x a(x)$ is nondecreasing and concave, and $\sum_{n=1}^{\infty} n^{-1} a(n)<\infty$.

Remark A.1. By applying Lemma A.1, we find that the function $a(x)$ described in Lemma A.2 is such that, for every $\alpha, 1<\alpha \leq 2, x a^{\alpha}\left(x^{1 / \alpha}\right)$ is concave for $x>1$. Indeed, the function $a(x)$ is defined for all $x \geq 0$ as

$$
a(x):=a_{1} 1_{[0,1)}(x)+x^{-1}\left(a_{1}+\int_{1}^{x} a_{\lfloor t\rfloor} \mathrm{d} t\right) 1_{[1, \infty)}(x),
$$

and, therefore,

$$
x a^{\alpha}\left(x^{1 / \alpha}\right)=\left(a_{1}+\int_{1}^{x^{1 / \alpha}} a_{\lfloor t\rfloor} \mathrm{d} t\right)^{\alpha}
$$

for all $x \geq 1$.

Lemma A.3. Let $f(x)$ be a positive, nonincreasing function on $\mathbb{R}_{+}$such that $\sum_{n=1}^{\infty} n^{-1} f(n)<$ $\infty$ and $x f(x)$ is nondecreasing, and let $\left\{a_{n}\right\}$ be a sequence of positive numbers such that $\left|a_{n+1}-a_{n}\right| \leq a_{n} f\left(a_{n} b^{n}\right)$ for some $b>1$. Then

$$
\lim _{n \rightarrow \infty} a_{n}=a<\infty .
$$

Moreover, there exists a value $z_{0}$, which depends on the function $f(x)$ and on $b$, such that $a>0$ if $a_{0}>z_{0}$.

Lemma A.4. Let $\left\{a_{n}\right\}_{n \geq 0}$ and $\left\{b_{n}\right\}_{n \geq 0}$ be sequences of nonnegative real numbers such that $a_{n+1}<c a_{n}+b_{n}, n \geq 0$, where $0<c<1$. If $\sum_{n=0}^{\infty} b_{n}<\infty$ then $\sum_{n=0}^{\infty} a_{n}<\infty$.

We refer the reader to [4] for the proof of the Lemmas A.2 and A.4, and to [2] for the proof of Lemma A.3.

\section{Acknowledgements}

The authors would like to thank the anonymous referee for interesting and helpful comments, which improved the paper. This research was supported by the Ministerio de Ciencia y Tecnología and the FEDER through the Plan Nacional de Investigación Científica, Desarrollo e Innovación Tecnológica, grant no. BFM2003-06074.

\section{References}

[1] González, M., Martínez, R. and Mota, M. (2005). On the unlimited growth of a class of homogeneous multitype Markov chains. Bernoulli 11, 559-570.

[2] Klebaner, F. (1984). Geometric rate of growth in population-size-dependent branching processes. J. Appl. Prob. 21, 40-49.

[3] Klebaner, F. (1985). A limit theorem for population-size-dependent branching processes. J. Appl. Prob. 22, 48-57.

[4] Klebaner, F. (1989). Geometric growth in near-supercritical population size dependent multitype Galton-Watson processes. Ann. Prob. 17, 1466-1477.

[5] Mode, C. J. (1971). Multitype Branching Processes. Elsevier, New York.

[6] Seneta, E. (1981). Nonnegative Matrices and Markov Chains, 2nd edn. Springer, New York.

[7] Sevast'Janov, B. A. And Zubkov, A. M. (1974). Controlled branching processes. Theor. Prob. Appl. 19, 14-24.

[8] Von Bahr, B. And Esseen, C. G. (1965). Inequalities for the $r$ th absolute moment of a sum of random variables. Anal. Math. Statist. 36, 299-303. 